\title{
Role of the Tunneling Ray in Near-Critical-Angle Scattering by a Dielectric Sphere
}

James A. Lock

Cleveland State University, j.lock@csuohio.edu

Follow this and additional works at: https://engagedscholarship.csuohio.edu/sciphysics_facpub

Part of the Physics Commons

How does access to this work benefit you? Let us know!

\section{Publisher's Statement}

This paper was published in Journal of the Optical Society of America A: Optics Image Science and Vision and is made available as an electronic reprint with the permission of OSA. The paper can be found at the following URL on the OSA website: http://www.opticsinfobase.org/josaa/ abstract.cfm?URI=josaa-20-3-499. Systematic or multiple reproduction or distribution to multiple locations via electronic or other means is prohibited and is subject to penalties under law.

\section{Original Citation}

Lock, James A. "Role of the Tunneling Ray in Near-Critical-Angle Scattering by a Dielectric Sphere." Journal of the Optical Society of America A: Optics Image Science and Vision 20 (2003): 499-507.

\section{Repository Citation}

Lock, James A., "Role of the Tunneling Ray in Near-Critical-Angle Scattering by a Dielectric Sphere" (2003). Physics Faculty Publications. 71.

https://engagedscholarship.csuohio.edu/sciphysics_facpub/71

This Article is brought to you for free and open access by the Physics Department at EngagedScholarship@CSU. It has been accepted for inclusion in Physics Faculty Publications by an authorized administrator of EngagedScholarship@CSU. For more information, please contact library.es@csuohio.edu. 


\title{
Role of the tunneling ray in near-critical-angle scattering by a dielectric sphere
}

\author{
James A. Lock \\ Department of Physics, Cleveland State University, Cleveland, Ohio 44115
}

Received July 15, 2002; revised manuscript received October 3, 2002; accepted October 9, 2002

\begin{abstract}
The scattering far zone for light transmitted through a sphere following $p-1$ internal reflections by a family of near-grazing incident rays is subdivided into a lit region and a shadow region. The sharpness of the ray theory transition between the lit and the shadow regions is smoothed in wave theory by radiation shed by electromagnetic surface waves. It is shown that when higher-order terms in the physical optics approximation to the phase of the partial-wave scattering amplitudes are included, the transition between the lit and the shadow regions becomes a two-ray-to-zero-ray transition, called a superweak caustic in analogy to the more familiar scattering caustics and weak scattering caustics. One of the merged rays is a tunneling ray. (c) 2003 Optical Society of America
\end{abstract}

OCIS codes: $080.1510,290.0290,290.4020$.

\section{INTRODUCTION}

When light is scattered by a large particle possessing a high degree of symmetry, such as a sphere, spheroid, or circular cylinder, and the scattered light is examined in the context of ray theory, a partial focusing of the light rays called an optical caustic occurs on certain surfaces in the near and far zones. ${ }^{1}$ Although the scattered-ray intensity diverges on caustics, these divergences are softened in wave theory, where the scattered field remains large but finite. When the observation point crosses a caustic, the number of geometrical rays contributing to the field changes by an even integer. For example, the number of contributing rays changes from two to zero for a fold caustic (i.e., a rainbow), from three to one for a cusp caustic (i.e., a cylindrical-aberration caustic), and from four to zero for the focal section of a hyperbolic umbilic caustic (which occurs in scattering by an oblate spheroid $^{2,3}$ ). The large wave intensity on a caustic is accompanied on either one or both sides by an interference pattern when two or more rays contribute to the wave field there.

Other scattering structures, known as weak caustics, occur in ray theory when the scattered field is everywhere finite while its first derivative diverges on certain surfaces in the near and far zones. ${ }^{4}$ Again these divergences are smoothed in wave theory. As an example, a weak caustic occurs at the transition between total external reflection and partial external reflection when light is specularly reflected by a spherical air bubble in water. In this case, one geometrical ray passes through any given point on either side of the transition, producing a change of zero in the number of contributing rays when crossing the weak caustic. Nonetheless, an interference pattern appears in the total-external-reflection region produced by superposition of the reflected geometrical ray and light diffracted by the end point of the total-externalreflection region. ${ }^{5}$

A scattering structure of a different type occurs when a family of rays has near-grazing incidence on a dielectric sphere. The far zone consists of a lit region containing rays refracted into the sphere and making $p-1$ internal reflections before refracting back out and a shadow region into which no such rays are deflected. The scattering angle at the boundary between the lit and the shadow regions is called the critical scattering angle since a geometrical light ray scattered in that direction refracts into the sphere at the critical angle for total internal reflection. In ray theory the scattered field and its first derivative are finite at the critical scattering angle, while the second derivative of the field diverges there. In wave theory this transition is smoothed by radiation shed into the shadow region by electromagnetic surface waves created by the near-grazing incident rays. ${ }^{6}$ In the vicinity of the critical scattering angle the scattered-wave field is approximately proportional to a modified Fock function that interpolates smoothly between the geometrical optics field in the lit region and the surface-wave radiation in the shadow region. 6,7 As a result, this transition between the lit and the shadow regions is called a Fock transition, and the number of geometrical rays contributing to the field at any point changes from one to zero when the rays cross the critical scattering angle.

On the basis of the ray theory divergence of the second derivative of the field at the critical scattering angle, one is tempted to classify the Fock transition as a superweak caustic in analogy to the ray theory divergences giving rise to caustics and weak caustics. In this paper we shall explore this interpretation of the Fock transition for scattering of a plane wave by a dielectric spherical particle in a vacuum. In particular, we find that when higher orderterms are included in the physical optics approximation to the phase of the Mie scattered field, a two-ray-to-zeroray transition is found to occur at the critical scattering angle. The two rays in the lit region are the near-grazing incident geometrical ray and a tunneling ray $^{8}$ whose impact parameter with respect to the sphere is larger than the sphere's radius. Even though this second ray classi- 
cally misses the sphere, a small percentage of its amplitude is captured by the centrifugal barrier surrounding the sphere and tunnels through it to the sphere's surface. The ray then refracts inside, makes $p-1$ internal reflections, refracts back outside, tunnels back out through the centrifugal barrier, and then freely propagates to the far zone as a scattered ray. Since the amplitude of the second ray is greatly reduced by the two tunnelings through the centrifugal barrier, its interference with the geometrical ray in the lit region is exceedingly weak.

The body of this paper is organized as follows. In Section 2 I briefly describe scattering of near-grazing incident geometrical rays by a sphere. In Section 3 I review both the Debye series expansion of Mie theory and the proportionality of the scattering amplitudes in the vicinity of the critical scattering angle to a modified Fock function in the context of the usual physical optics approximation. The new results are contained in Sections 4 and 5 . In Section $4 \mathrm{I}$ include the next higher-order term in the series expansion of the phase of the Debye series partialwave transmission and reflection amplitudes beyond that of the usual physical optics approximation. We find that in the lit region the phase now possesses a second stationary-phase point corresponding to a geometrical ray whose impact parameter is greater than the sphere radius, which we physically interpret as the tunneling ray. ${ }^{9}$ In Section $5 \mathrm{I}$ verify the existence of the second stationary-phase point by numerically determining the relation between the scattering angle and the partialwave number of the tunneling ray in the exact Debye partial-wave scattering amplitudes. Finally, conclusions are summarized in Section 6.

\section{SCATTERING OF NEAR-GRAZING INCIDENT RAYS BY A SPHERE}

A family of parallel rays with amplitude $E_{0}$, wavelength $\lambda$, wave number $k=2 \pi / \lambda$, and angular frequency $\omega$, polarized in the $x$ direction and traveling in the $z$ direction, is incident on a sphere of radius $a$ and refractive index $n>1$ whose center is at the origin of coordinates. Light scattered by the sphere consists of an infinite number of ray families parameterized by the integer $p$ with $0 \leqslant p$ $<\infty$. Specularly reflected rays together with the diffracted field are known as the $p=0$ scattered-ray family. The family of $p \geqslant 1$ scattered rays refracts into the sphere and makes $p-1$ internal reflections before refracting back out. If $\theta_{i}$ is the angle an incoming ray makes with the normal to the sphere surface and $\theta_{t}$ is the angle the refracted ray makes with the normal, the deflection angle $\Theta$ of the corresponding scattered ray in the $p$ family is

$$
\Theta=(p-1) \pi+2 \theta_{i}-2 p \theta_{t},
$$

where

$$
\sin \left(\theta_{i}\right)=n \sin \left(\theta_{t}\right) .
$$

In the $r \rightarrow \infty$ far-zone limit, the electric field of the scattered $p$-ray family is

$$
\begin{aligned}
\mathbf{E}_{\mathrm{scatt}}^{(p)}(\mathbf{r}, t)= & -\left(i E_{0} / k r\right) \exp (i k r-i \omega t) \\
& \times\left[-S_{\mathrm{TM}}^{(p)}(\theta) \cos (\phi) \mathbf{u}_{\theta}+S_{\mathrm{TE}}^{(p)}(\theta) \sin (\phi) \mathbf{u}_{\phi}\right],
\end{aligned}
$$

where $\theta$ and $\phi$ are the spherical coordinate system angles and TE and TM denote the transverse electric and transverse magnetic polarizations of the scattered light. The ray scattering amplitudes $S_{\alpha}^{(p)}(\theta)$ for $p \geqslant 1$ and $\alpha=\mathrm{TE}$ or TM are ${ }^{10}$

$$
\begin{aligned}
S_{\alpha}^{(p)}(\theta)= & x\left[\sin \left(\theta_{i}\right) \cos \left(\theta_{i}\right) / 2 \sin (\theta)\right]^{1 / 2} \\
& \times\left|1-\left[p \cos \left(\theta_{i}\right) / n \cos \left(\theta_{t}\right)\right]\right|^{-1 / 2} t_{\alpha}^{21}\left(\theta_{i}\right) \\
& \times\left[r_{\alpha}^{11}\left(\theta_{i}\right)\right]^{p-1} t_{\alpha}^{12}\left(\theta_{i}\right) \\
& \times \exp \left\{2 i x\left[p n \cos \left(\theta_{t}\right)-\cos \left(\theta_{i}\right)\right]+i \xi_{p}\right\},
\end{aligned}
$$

where the size parameter of the sphere is

$$
x \equiv k a \text {. }
$$

The flat-surface electric field Fresnel coefficients ${ }^{11}$ for refraction into the sphere, internal reflection, and refraction out of the sphere are $t_{\alpha}^{21}\left(\theta_{i}\right), r_{\alpha}^{11}\left(\theta_{i}\right)$, and $t_{\alpha}^{12}\left(\theta_{i}\right)$, respectively, and $\xi_{p}$ is the phase shift incurred by the scattered ray as a result of crossing focal lines. ${ }^{12}$ The deflection angle $\Theta$ and scattering angle $\theta$ of an outgoing ray are related by

$\theta=\left\{\begin{array}{ll}\Theta-2 \pi N & \text { if } 2 \pi N \leqslant \Theta \leqslant 2 \pi N+\pi \\ 2 \pi(N+1)-\Theta & \text { if } 2 \pi N+\pi<\Theta<2 \pi(N+1)\end{array}\right.$.

We are particularly interested in rays with neargrazing incidence on the sphere, i.e.,

$$
\sin \left(\theta_{i}\right)=1-\delta,
$$

with $\delta \ll 1$. These rays are only weakly transmitted into the sphere, since

$$
t_{\alpha}^{21}\left(\theta_{i}\right) t_{\alpha}^{12}\left(\theta_{i}\right)=2^{5 / 2} \beta^{2}\left[\delta /\left(n^{2}-1\right)\right]^{1 / 2}+O(\delta),
$$

where

$$
\beta= \begin{cases}1 & \text { for } \mathrm{TE} \\ n & \text { for } \mathrm{TM}\end{cases}
$$

But once inside the sphere they make many internal reflections before attenuating, since

$$
r_{\alpha}^{11}\left(\theta_{i}\right)=1-O\left(\delta^{1 / 2}\right)
$$

The deflection angle of a scattered near-grazing ray is

$$
\Theta=\Theta_{c}^{p}-2^{3 / 2} \delta^{1 / 2}+O(\delta),
$$

where

$$
\Theta_{c}^{p}=2 p \arccos (1 / n)
$$

is the critical deflection angle of the ray incident with $\theta_{i}=\pi / 2$. No rays in the $p$ family are scattered with $\Theta>\Theta_{c}^{p}$. If we substitute Eqs. (7), (8), (10), and (11) into Eq. (4), the scattering amplitudes of the $p \geqslant 1$ neargrazing rays become 


$$
\begin{aligned}
S_{\alpha}^{(p)}(\theta)= & \beta^{2} x\left(\Theta_{c}^{p}-\Theta\right)^{3 / 2}\left[\left(n^{2}-1\right) \sin (\theta)\right]^{-1 / 2} \\
& \times \exp \left[2 i p x\left(n^{2}-1\right)^{1 / 2}-i x\left(\Theta_{c}^{p}-\Theta\right)+i \xi_{p}\right],
\end{aligned}
$$

where

$$
\xi_{p}=\left\{\begin{array}{l}
\exp [-i \pi p / 2-i \pi(N+1)] \\
\text { if } 2 \pi N \leqslant \Theta \leqslant 2 \pi N+\pi \\
\exp [-i \pi(p+1) / 2-i \pi(N+1)] \\
\text { if } 2 \pi N+\pi<\Theta<2 \pi(N+1)
\end{array} .\right.
$$

Although the scattered electric field and its first derivative with respect to $\Theta$ are finite at $\Theta=\Theta_{c}^{p}$, the second derivative diverges as $\Theta$ approaches $\Theta_{c}^{p}$ from below.

\section{MIE THEORY AND THE FOCK TRANSITION FOR NEAR-GRAZING INCIDENCE}

Mie theory smooths the transition between the lit region $\Theta<\Theta_{c}^{p}$ and the shadow region $\Theta>\Theta_{c}^{p}$. For a plane wave polarized in the $x$ direction, traveling in the $z$ direction, and incident on the sphere, the far-zone Mie scattered electric field is

$$
\begin{aligned}
\mathbf{E}_{\mathrm{scat}}(\mathbf{r}, t)= & -i\left(E_{0} / k r\right) \exp (i k r-i \omega t)\left[-S_{2}(\theta)\right. \\
& \left.\times \cos (\phi) \mathbf{u}_{\theta}+S_{1}(\theta) \sin (\phi) \mathbf{u}_{\phi}\right],
\end{aligned}
$$

where the scattering amplitudes $S_{j}(\theta)$ for $j=1,2$ are

$$
\begin{aligned}
& S_{1}(\theta)=\sum_{l=1}^{\infty}[(2 l+1) / l(l+1)]\left[a_{l} \pi_{l}(\theta)+b_{l} \tau_{l}(\theta)\right], \\
& S_{2}(\theta)=\sum_{l=1}^{\infty}[(2 l+1) / l(l+1)]\left[a_{l} \tau_{l}(\theta)+b_{l} \pi_{l}(\theta)\right],
\end{aligned}
$$

with $l$ being the partial wave number. ${ }^{13}$ The partialwave scattering amplitudes $a_{l}$ and $b_{l}$ are ratios of sums of products of spherical Bessel functions, spherical Neumann functions, and their derivatives whose explicit form is given in Ref. 13, and the angular functions $\pi_{l}(\theta)$ and $\tau_{l}(\theta)$ are related to associated Legendre polynomials. The connection between Mie theory and ray theory for scattering by large spheres, $x \gg 1$, uses the Debye series decomposition of the partial-wave scattering amplitudes $6,8,14$

$$
\left.\begin{array}{l}
a_{l} \\
b_{l}
\end{array}\right\}=(1 / 2)\left[1-R_{l}^{22}-\sum_{p=1}^{\infty} T_{l}^{21}\left(R_{l}^{11}\right)^{p-1} T_{l}^{12}\right],
$$

where the first term inside the square brackets in Eq. (18) corresponds to diffraction, $R_{l}^{22}$ is the complex amplitude for specular reflection of the radially incoming partial wave $l$ by the sphere surface, $T_{l}^{21}$ is the complex amplitude for transmission of the partial wave into the sphere, and $R_{l}^{11}$ and $T_{l}^{12}$ are the complex amplitudes for internal reflection and transmission out of the sphere. These amplitudes are again ratios of sums of products of spherical Bessel functions, spherical Neumann functions, and their derivatives whose explicit form is given in Ref. 14 .
According to van de Hulst's localization principle, ${ }^{15}$ we are interested primarily in partial waves in the so-called edge region, ${ }^{6}$

$$
l+(1 / 2)=x+z(x / 2)^{1 / 3},
$$

with $-5 \lessgtr z \lesssim 5$, corresponding to the near-grazing incident rays of Eq. (7). The characteristic width of the edge region is $(x / 2)^{1 / 3}$, and the $z$ measures the distance of the ray in from or out beyond the sphere radius. The spherical Bessel and spherical Neumann functions appearing in the various terms of Eq. (18) for partial waves in the edge region are approximated by ${ }^{16}$

$$
\begin{aligned}
& j_{l}(x) \approx \pi^{1 / 2} A i(z) /\left(2^{1 / 6} x^{5 / 6}\right), \\
& n_{l}(x) \approx-\pi^{1 / 2} B i(z) /\left(2^{1 / 6} x^{5 / 6}\right), \\
& j_{l}^{\prime}(x) \approx-\pi^{1 / 2} 2^{1 / 6} A i^{\prime}(z) / x^{7 / 6}, \\
& n_{l}^{\prime}(x) \approx \pi^{1 / 2} 2^{1 / 6} B i^{\prime}(z) / x^{7 / 6},
\end{aligned}
$$

where $A i(z)$ is the Airy function ${ }^{17}$ and $B i(z)$ is the second solution of the Airy differential equation,

$$
\begin{aligned}
j_{l}(y) & \approx n^{1 / 2} \cos (Y) /\left[\left(n^{2}-1\right)^{1 / 4} y\right], \\
n_{l}(y) & \approx n^{1 / 2} \sin (Y) /\left[\left(n^{2}-1\right)^{1 / 4} y\right], \\
j_{l}^{\prime}(y) & \approx-\left(n^{2}-1\right)^{1 / 4} \sin (Y) /\left(n^{1 / 2} y\right), \\
n_{l}^{\prime}(y) & \approx\left(n^{2}-1\right)^{1 / 4} \cos (Y) /\left(n^{1 / 2} y\right), \\
y & =n x,
\end{aligned}
$$

and

$$
\begin{aligned}
Y= & -\pi / 4+n x\left\{1-\left[1+(z / 2)(2 / x)^{2 / 3}\right]^{2} / n^{2}\right\}^{1 / 2} \\
& -x\left[1+(z / 2)(2 / x)^{2 / 3}\right] \arcsin (\{1-[1+(z / 2) \\
& \left.\left.\left.\times(2 / x)^{2 / 3}\right]^{2} / n^{2}\right\}^{1 / 2}\right) .
\end{aligned}
$$

The series expansion of the phase $Y$ of Eq. (23) in decreasing powers of $x$ is rapidly convergent for $x \gg 1$. The first three terms of the expansion are

$$
\begin{aligned}
Y \approx- & \pi / 4+x\left(n^{2}-1\right)^{1 / 2}-x \arccos (1 / n)-z(x / 2)^{1 / 3} \\
& \times \arccos (1 / n)+\left(z^{2} / 4\right)(x / 2)^{-1 / 3}\left(n^{2}-1\right)^{-1 / 2} .
\end{aligned}
$$

Substituting Eqs. (20) and (21) into the partial-wave transmission and reflection coefficients of Eq. (18), substituting the appropriate Debye term for $a_{l}$ and $b_{l}$ into the scattering amplitudes $S_{j}(\theta)$ of Eqs. (16) and (17), which we now denote as $S_{j}^{(p)}(\theta)$, converting the sum over partial waves in the scattering amplitudes into an integral $^{18,19}$ over $z$, using the fact that ${ }^{20} \tau_{l}(\theta) \gg \pi_{l}(\theta)$ for $l \gg 1$ when $\theta$ is not near 0 or $\pi$, using the asymptotic expression for $\tau_{l}(\theta)$, and retaining only the leading term in $x$ in the magnitude of $S_{j}^{(p)}(\theta)$, we obtain after much algebra

$$
\begin{aligned}
S_{j}^{(p)}(\theta) \approx & \beta^{2}(8 x)^{1 / 2}\left[\pi^{3}\left(n^{2}-1\right) \sin (\theta)\right]^{-1 / 2} \\
& \times \int_{-\infty}^{\infty} \mathrm{d} z[B i(z)+i \operatorname{Ai}(z)]^{-2} \exp [2 i p Y+i x \Theta \\
& \left.+i z \Theta(x / 2)^{1 / 3}+i \pi p / 2+3 \pi i / 4+i \xi_{p}\right] .
\end{aligned}
$$

The mathematically precise way to convert the sum over partial waves into an integral is to use the Poisson sum- 
mation formula. ${ }^{6,21}$ The prescription used in Refs. 18 and 19 as well as here includes only the type-I class of surface waves ${ }^{22}$ that have circumnavigated the sphere less than one time, whereas the prescription of Refs. 6 and 21 includes all classes of surface waves for $p \geqslant 2$ that have circumnavigated the sphere all numbers of times. The result of relation (25), though approximate, is sufficient for our purposes. Including the terms in the series expansion of the phase $Y$ of relation (24) as small as $(x / 2)^{1 / 3}$ in relation (25), converting the integral to a contour integral in the complex $z$ plane, and writing the result in terms of the modified Fock function ${ }^{6,7}$

$$
\begin{aligned}
f(s)= & (1 / 2 \pi) \exp (i \pi / 6) \\
& \times \int_{\Gamma} \mathrm{d} z\{\operatorname{Ai}[z \exp (2 \pi i / 3)]\}^{-2} \exp (i s z),
\end{aligned}
$$

where $\Gamma$ is the path in the complex $z$ plane described in Fig. 10 of Ref. 7, we obtain

$$
\begin{aligned}
S_{j}^{(p)}(\theta) \approx & \beta^{2}\left[2 x / \pi\left(n^{2}-1\right) \sin (\theta)\right]^{1 / 2} \\
& \times \exp \left[2 i p x\left(n^{2}-1\right)^{1 / 2}+i x\left(\Theta-\Theta_{c}^{p}\right)\right. \\
& \left.+i \pi / 4+i \xi_{p}\right] f\left[\left(\Theta-\Theta_{c}^{p}\right)(x / 2)^{1 / 3}\right] .
\end{aligned}
$$

For $s \ll 0$, or equivalently $\Theta \ll \Theta_{c}^{p}$, when the Airy function in Eq. (26) is replaced by its oscillatory asymptotic form ${ }^{23}$ the resulting integral has a single stationary-phase point at

$$
z=-s^{2} / 4
$$

and may be evaluated by means of the method of stationary phase to give 6

$$
f(s) \approx \pi^{1 / 2}|s|^{3 / 2} \exp \left(-i \pi / 4-i s^{3} / 12\right) .
$$

Substituting relation (29) into relation (27) produces the ray theory result of Eq. (13) with the identifications $S_{1}^{(p)}(\theta)=S_{\mathrm{TE}}^{(p)}(\theta)$ and $S_{2}^{(p)}(\theta)=S_{\mathrm{TM}}^{(p)}(\theta)$; i.e., each Mie scattering amplitude corresponds to a single polarization of the scattered rays for large $x$. If we substitute the argument of the Fock function in relation (27) into Eq. (28), the stationary phase point occurs for

$$
z=-(1 / 4)\left(\Theta_{c}^{p}-\Theta\right)^{2}(x / 2)^{2 / 3}
$$

or

$$
l+(1 / 2)=x-x\left(\Theta_{c}^{p}-\Theta\right)^{2} / 8 .
$$

This stationary-phase partial-wave number corresponding to the refracted geometrical ray is independent of polarization and has no dependence on $p$ or $n$ other than that implicit in $\Theta_{c}^{p}$.

For $s \gg 0$, or equivalently $\Theta \gg \Theta_{c}^{p}$, the integral in Eq. (26) may be evaluated by the rapidly convergent residue series of the second-order poles of the integrand to give ${ }^{6}$

$$
\begin{aligned}
f(s) \approx & s \exp (-i \pi / 6) \sum_{n}\left[\mathrm{Ai}^{\prime}\left(-w_{n}\right)\right]^{-2} \\
& \times \exp \left[\left(-3^{1 / 2} s w_{n} / 2\right)+i\left(s w_{n} / 2\right)\right],
\end{aligned}
$$

where $-w_{n}$ are the zeros of the Airy function ${ }^{24}$

$$
\operatorname{Ai}\left(-w_{n}\right)=0 .
$$

Substitution of relation (32) into relation (27) describes the contribution to the scattering amplitude of radiation shed into the shadow region by electromagnetic surface waves:

$$
\begin{aligned}
S_{j}^{(p)}(\theta) \approx & \beta^{2} 2^{1 / 6} x^{5 / 6}\left[\pi\left(n^{2}-1\right) \sin (\theta)\right]^{-1 / 2} \\
& \times \exp \left[2 i p x\left(n^{2}-1\right)^{1 / 2}\right. \\
& \left.+i x\left(\Theta-\Theta_{c}^{p}\right)+i \pi / 12+i \xi_{p}\right] \\
& \times\left(\Theta-\Theta_{c}^{p}\right) \sum_{n}\left[\operatorname{Ai}^{\prime}\left(-w_{n}\right)\right]^{-2} \\
& \times \exp \left[i\left(w_{n} / 2\right)\left(\Theta-\Theta_{c}^{p}\right)(x / 2)^{1 / 3}\right] \\
& \times \exp \left[-\left(3^{1 / 2} w_{n} / 2\right)\left(\Theta-\Theta_{c}^{p}\right)(x / 2)^{1 / 3}\right] .
\end{aligned}
$$

The Fock function shown in Fig. 1 smoothly joins the geometrical ray amplitude in the lit region, Eq. (13), and the surface-wave radiation in the shadow region, relation

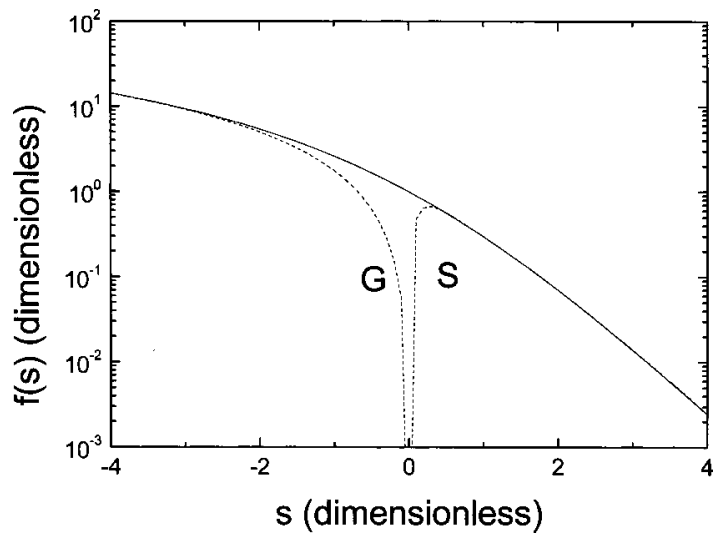

Fig. 1. Magnitude of the modified Fock function $f(s)$ of Eq. (26) numerically computed by using the contour of Fig. 10 of Ref. 7 on which the integrand damps most rapidly to zero as a function of the integration variable $z$. The dashed curve $\mathrm{G}$ is the geometrical-ray stationary-phase limit of relation (29), and the dashed curve $\mathrm{S}$ is the surface-wave limit of relation (32) obtained by using the first five zeros of the Airy function.

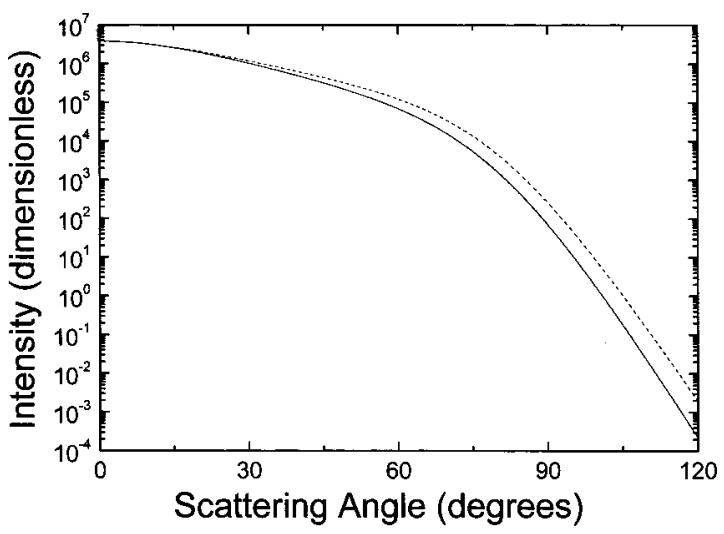

Fig. 2. Debye intensity for transmission, $p=1$, by a sphere with size parameter $x=1000.0$ and refractive index $n=1.333$ as a function of scattering angle for the amplitudes of Eqs. (16) and (17): $j=1$, (solid curve) and $j=2$ (dashed curve). The scattering angle $\theta_{c}^{1}=82.79^{\circ}$ separates the lit region from the shadow region in ray theory. The Debye intensity smoothes the transition between these two regions. 
(34). This behavior is also apparent in the computed $p=1$ Debye intensity shown in Fig. 2. As the size parameter of the sphere increases, the amplitude of the surface-wave radiation increases more slowly than the geometric optics amplitude, and the surface-wave radiation attenuates over a decreasing angular interval, thus approaching the geometrical optics description of the transition as $x \rightarrow \infty$.

\section{CONTRIBUTION OF THE TUNNELING RAY TO NEAR-GRAZING-INCIDENCE SCATTERING}

As mentioned previously, when the terms in the series expansion of the phase $Y$ of Eq. (23) as small as $(x / 2)^{1 / 3}$ are substituted into relation (25) and the oscillatory asymptotic form of $[B i(z)+i \operatorname{Ai}(z)]^{-2}$ is used, the resulting integral for $\Theta<\Theta_{c}^{p}$ has a single stationary-phase point for $z<0$ corresponding to a geometrical ray that enters the sphere and re-emerges following $p-1$ internal reflections. No stationary-phase point occurs for $\Theta$ $>\Theta_{c}^{p}$. The situation changes, however, if the term of or$\operatorname{der}(x / 2)^{-1 / 3}$ in $Y$ is now also included in relation (25). For $\Theta<\Theta_{c}^{p}$ the location of the $z<0$ geometrical-ray stationary point changes only slightly. But now a new $z>0$ stationary-phase point corresponding to a ray incident beyond the edge of the sphere occurs at

$$
z=\left[\left(n^{2}-1\right)^{1 / 2} / p\right]\left(\Theta_{c}^{p}-\Theta\right)(x / 2)^{2 / 3},
$$

or

$$
l+(1 / 2)=x+x\left(n^{2}-1\right)^{1 / 2}\left(\Theta_{c}^{p}-\Theta\right) / 2 p .
$$

Again, no stationary-phase point occurs for $\Theta>\Theta_{c}^{p}$. The partial-wave number of the second stationary-phase point in the lit region is polarization independent and increases more slowly for large $p$ than it does for small $p$. When the new stationary point satisfies $z \gtrsim 1$, the quantity $[B i(z)+i \operatorname{Ai}(z)]^{-2}$ in relation (25) can be approximated by $[\operatorname{Bi}(z)]^{-2}$. If we substitute the exponential asymptotic form $^{25}$ of $\mathrm{Bi}(z)$, a stationary-phase evaluation of the integral for $\Theta<\Theta_{c}^{p}$ including both stationary points gives

$$
\begin{aligned}
S_{j}^{(p)}(\theta) \approx & \beta^{2} x\left[\left(n^{2}-1\right) \sin (\theta)\right]^{-1 / 2} \\
& \times \exp \left[2 i p x\left(n^{2}-1\right)^{1 / 2}-i x\left(\Theta_{c}^{p}-\Theta\right)+i \xi_{p}\right] \\
& \times\left\{\left(\Theta_{c}^{p}-\Theta\right)^{3 / 2}-\left(\Theta_{c}^{p}-\Theta\right)^{1 / 2}\right. \\
& \times\left[2^{3 / 2}\left(n^{2}-1\right)^{1 / 2} / p\right] \\
& \times \exp \left[-i x\left(n^{2}-1\right)^{1 / 2}\left(\Theta_{c}^{p}-\Theta\right)^{2} / 4 p\right] \\
& \left.\times \exp \left[-2 x\left(n^{2}-1\right)^{3 / 4}\left(\Theta_{c}^{p}-\Theta\right)^{3 / 2} / 3 p^{3 / 2}\right]\right\} .
\end{aligned}
$$

The physical interpretation of the second stationaryphase point in relation (37) in terms of a straightforward extension of ray theory is as follows. In Fig. 3 for the case of $p=1$, two different rays incident on the sphere in the edge region exit with the same scattering angle $\theta$ such that $\Theta<\Theta_{c}^{p}$. The first is the usual geometrical ray whose impact parameter is

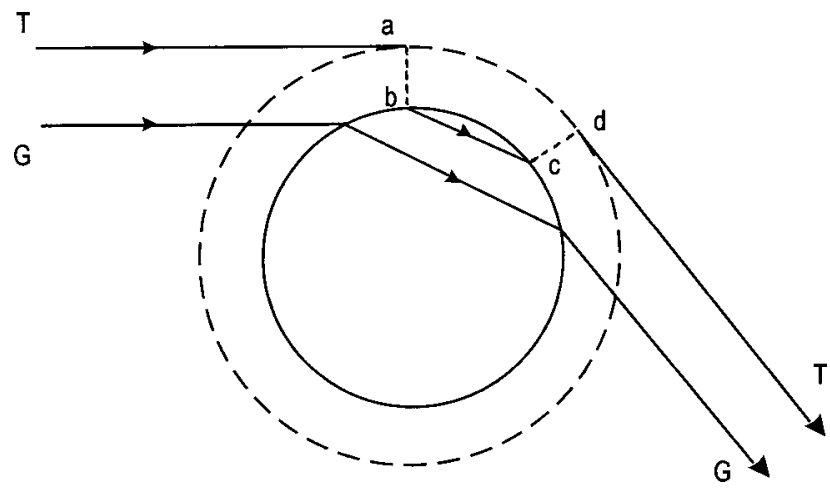

Fig. 3. Trajectory of the $p=1$ geometrical ray $\mathrm{G}$ and the tunneling ray $\mathrm{T}$ with the same scattering angle. The extent of the centrifugal barrier surrounding the sphere and probed by the tunneling ray is indicated by the dashed line. The ray tunnels from $a$ to $b$ and from $c$ to $d$ through the centrifugal barrier.

$$
b=a \sin \left(\theta_{i}\right)=a-\delta a,
$$

where $\delta \ll 1$, and $\delta$ is related to the angular distance from the critical scattering angle $\Theta_{c}^{p}-\Theta$ by Eq. (11). The ray's optical path length with respect to that of a suitably chosen reference ray is

$$
\begin{aligned}
\Phi_{\mathrm{opl}} & =2 x\left[p n \cos \left(\theta_{t}\right)-\cos \left(\theta_{i}\right)\right] \\
& =2 p x\left(n^{2}-1\right)^{1 / 2}-x\left(\Theta_{c}^{p}-\Theta\right),
\end{aligned}
$$

as in Eq. (13), and the partial wave corresponding to the geometrical ray is given by Eq. (31) in agreement with van de Hulst's localization principle.

The contribution to relation (37) of the second stationary point of Eq. (35) is associated with the tunneling ray of Fig. 3. The tunneling ray passes the sphere with an impact parameter

$$
b=a \sin \left(\theta_{i}\right)=a+\Delta a,
$$

where $\Delta \ll 1$. Classically, this ray misses the sphere. But in wave theory a small portion of it, $A_{\alpha}(\Delta)$, is captured by the centrifugal barrier surrounding the sphere and tunnels through it to the sphere surface. ${ }^{26}$ The angle of incidence of the ray at the sphere surface implicit in Eq. (40) is

$$
\theta_{i} \approx \pi / 2-i(2 \Delta)^{1 / 2},
$$

and the angle of refraction inside the sphere, $\theta_{t}$, is given by Snell's law:

$$
\theta_{t} \approx \arcsin (1 / n)+\Delta /\left(n^{2}-1\right)^{1 / 2} .
$$

This refracted angle is larger than that of the critical ray incident on the sphere with $\theta_{i}=\pi / 2$. The tunneling-ray amplitude refracted into the sphere is given by the flatsurface electric field Fresnel transmission coefficient $t_{\alpha}^{21}(\Delta)$. Evaluating $\cos \left(\theta_{i}\right)$ and $\cos \left(\theta_{t}\right)$ from relations (41) and (42) and substituting into the formula for the Fresnel coefficient, ${ }^{11}$ we obtain

$$
t_{\alpha}^{21}(\Delta) \approx 2 i \beta(2 \Delta)^{1 / 2}\left(n^{2}-1\right)^{-1 / 2}+O(\Delta) .
$$

When the ray inside the sphere next encounters the sphere surface, it internally reflects with the flat-surface electric field Fresnel coefficient

$$
r_{\alpha}^{11}(\Delta) \approx 1-O\left(\Delta^{1 / 2}\right)
$$


After $p-1$ internal reflections, it finally refracts back out of the sphere with the flat-surface Fresnel coefficient

$$
t_{\alpha}^{12}(\Delta) \approx 2 \beta+O\left(\Delta^{1 / 2}\right)
$$

and is damped by another factor of $A_{\alpha}(\Delta)$ as it tunnels back out through the centrifugal barrier. The ray is then reconverted into a geometrical ray and propagates out to the far zone. The deflection angle of the tunneling ray is found from relations (1), (41), and (42) to be

$$
\Theta=\Theta_{c}^{p}-2 p \Delta /\left(n^{2}-1\right)^{1 / 2},
$$

and the optical path length of the tunneling ray's trajectory is

$$
\Phi_{\mathrm{opl}}=2 p n x \cos \left(\theta_{t}\right)=2 p x\left(n^{2}-1\right)^{1 / 2}-x\left(\Theta_{c}^{p}-\Theta\right),
$$

which is identical to Eq. (39) for the geometrical ray scattered through the same angle.

When Eqs. (40) and (46) are combined with van de Hulst's localization principle, the partial wave corresponding to the tunneling ray is

$$
l+(1 / 2)=k b=x+x\left(n^{2}-1\right)^{1 / 2}\left(\Theta_{c}^{p}-\Theta\right) / 2 p,
$$

in agreement with Eq. (36). Evaluating the scattered intensity of the tunneling ray by means of flux conservation gives the ray amplitude as

$$
\begin{aligned}
S_{\alpha}^{(p)}(\theta)= & x[\sin (\theta)|\mathrm{d} \theta / \mathrm{d} \Delta|]^{-1 / 2} A_{\alpha}(\Delta) t_{\alpha}^{21}(\Delta) \\
& \times\left[r_{\alpha}^{11}(\Delta)\right]^{p-1} t_{\alpha}^{12}(\Delta) A_{\alpha}(\Delta) \exp \left(i \Phi_{\mathrm{opl}}+i \xi_{p}\right) .
\end{aligned}
$$

If we substitute Eqs. (43)-(47) into Eq. (49) with the identification

$$
\begin{aligned}
A_{\alpha}(\Delta) & \approx \exp (i \pi / 4) \exp \left[-x\left(n^{2}-1\right)^{3 / 4}\left(\Theta_{c}^{p}-\Theta\right)^{3 / 2} / 3 p^{3 / 2}\right] \\
& =\exp (i \pi / 4) \exp \left[-\Delta x(x / 2)^{1 / 3}\right] \\
& =\exp (i \pi / 4) \exp \left(-2 z^{3 / 2} / 3\right)
\end{aligned}
$$

for the tunneling amplitude, with $z$ as in Eq. (35), Eq. (49) is identical to the second term in relation (37) obtained from Mie theory. This result was obtained by extending ray theory to the tunneling ray of Fig. 3. The tunneling amplitude of relation (50) has the same functional dependence as the faster-than-exponential decay of the Airy function, which describes tunneling through a linearly increasing potential barrier of finite width. The centrifugal barrier surrounding the spherical particle has this same shape to first approximation. ${ }^{27,28}$ From Eq. (19) the nondimensional characteristic width of the centrifugal barrier surrounding the sphere is $(x / 2)^{1 / 3}$, and from Eq. (40) the nondimensional distance the ray must tunnel through the barrier to reach the sphere surface is $\Delta x$.

\section{NUMERICAL IDENTIFICATION OF THE TUNNELING RAY}

The analytical results of Section 4 required many approximations to the Debye series scattering amplitudes. In this section we investigate the behavior of the tunneling-ray stationary point by using the exact Debye partial-wave scattering amplitudes. We numerically compute the quantity

$$
D_{l}^{(p)}(\theta)=T_{l}^{21}\left(R_{l}^{11}\right)^{p-1} T_{l}^{12} \exp \left[i\left(l+\frac{1}{2}\right) \theta\right]
$$

as a function of $l$ for $p=1,2$, and $\theta$ in the vicinity of the critical scattering angle, and from the results we determine the partial wave number of each relative maximum and minimum of the phase of Eq. (51) as a function of scattering angle. The combination of partial-wave transmission and reflection amplitudes in Eq. (51) is the Debye contribution to $a_{l}$ and $b_{l}$ for a given value of $p$ as in Eq. (18). The complex exponential $\exp [i(l+1 / 2) \theta]$, along with $\exp [-i(l+1 / 2) \theta]$, which we do not consider since it provides negligible contribution for $p=1,2$, is proportional to the asymptotic limit of the angular function $\tau_{l}(\theta)$ in Eqs. (16) and (17) for large $l$. As a result, $D_{l}^{(p)}(\theta)$ is the dominant contribution to the summand of the Debye scattering amplitudes $S_{j}^{(p)}(\theta)$ for $j=1,2$.

The results for a sphere size parameter $x=1000.0$ and refractive index $n=1.333$, which approximates a $\sim 0.1$ mm-radius water droplet illuminated by visible light, are shown in Figs. 4-7. We shall first demonstrate that the stationary-phase points of Eq. (51) correspond to the impact parameter of the appropriate geometrical light rays. In Fig. 4 the stationary-phase partial-wave number is graphed as a function of scattering angle for transmission, $p=1$. This result is compared with the ray theory prediction for the incident-ray impact parameter as a function of scattering angle obtained with Eq. (1). The numerically obtained stationary-phase points were virtually independent of polarization, and the correspondence with ray theory is near perfect. This test calculation, along with that of Fig. 7 to be described below, demonstrates the validity of Eq. (51) in describing the summand of the Debye scattering amplitudes.

In Fig. 5 is graphed the partial-wave number of the $p=1$ geometrical and tunneling-ray stationary-phase points as a function of scattering angle near the critical

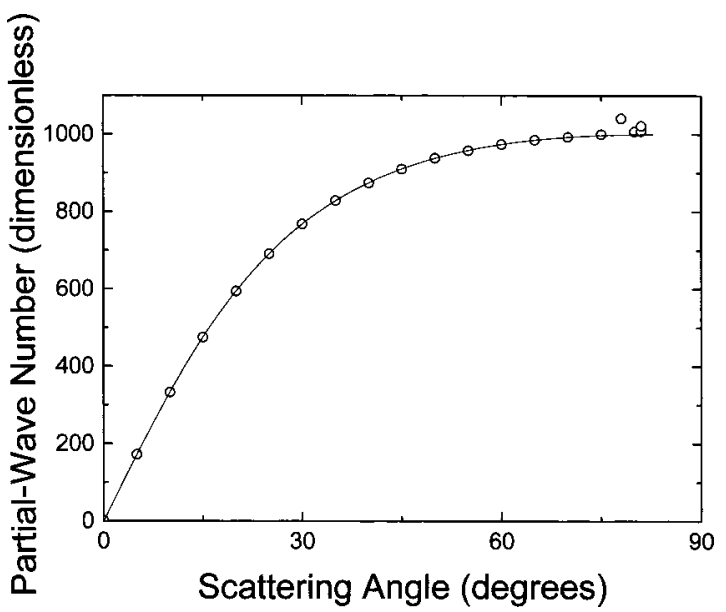

Fig. 4. Partial-wave number of the $p=1$ stationary-phase points of Eq. (51) as a function of scattering angle for $x=1000.0, n=1.333$, and $j=2$ (circles). The solid curve is the prediction of ray theory for the impact parameter of the transmitted geometrical ray. The the uppermost two open circles in the upper-right corner of the figure are tunneling-ray stationary-phase points. 


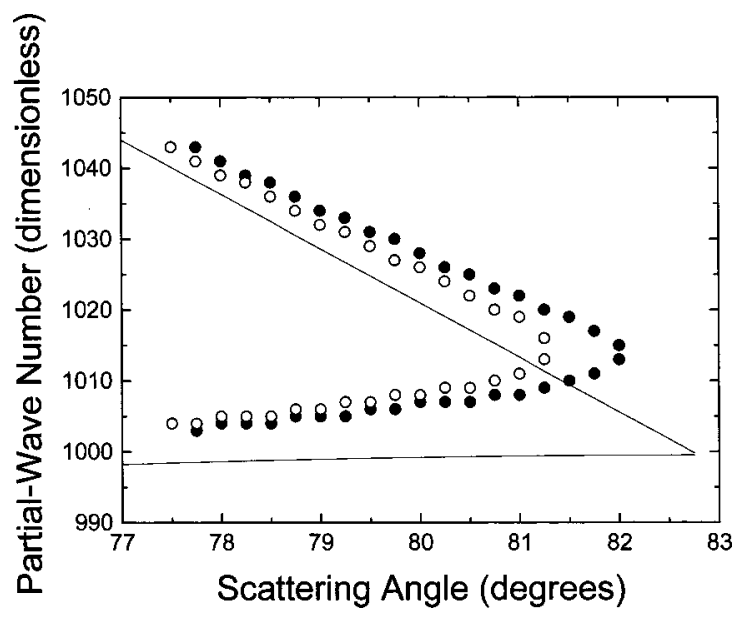

Fig. 5. Partial-wave number of the $p=1$ geometrical-ray and tunneling-ray stationary-phase points as a function of scattering angle for $x=1000.0$ and $n=1.333$. The solid circles are the stationary-phase points of the $j=1$ amplitude, and the open circles are the stationary-phase points of the $j=2$ amplitude. The lower and upper solid lines are the predictions of Eqs. (31) and (36), respectively.

scattering angle. This is a magnified version of the upper-right-hand corner of Fig. 4. The analytical predictions of Eqs. (31) and (36) are shown for comparison. The scattering angle of the numerically obtained stationary points is slightly larger than that predicted by the analytical approximations and is slightly polarization dependent. The stationary-phase points merge at a slightly larger partial wave than $x-1 / 2$, and the scattering angle of the merge is slightly smaller than the critical scattering angle. In spite of these small differences, the analytical formulas of Eqs. (31) and (36) provide a good firstorder approximation of the behavior of the stationaryphase points. Thus on the basis of this numerical computation with use of the exact Debye partial-wave scattering amplitudes, as well as on the basis of the analytical results of Section 4, the Fock transition may be interpreted as a two-ray-to-zero-ray transition. To illustrate the nature of the transition further, in Figs. 6(a)6(c) are graphed the phase of $D_{l}^{(p)}(\theta)$ for $p=1$ and $j=2$ as a function of partial-wave number for three scattering angles $79.5^{\circ}, 81.0^{\circ}$, and $82.75^{\circ}$ in the vicinity of the ray theory critical scattering angle $\theta_{c}^{1}=82.79^{\circ}$. The two stationary-phase points approach each other, merge, and vanish. Radiation is shed by electromagnetic surface waves into the shadow region to the right of the merge point of the two ray trajectories in Figs. 4 and 5, i.e., to larger scattering angles.

In Fig. 7 the partial-wave number of the $j=2$ stationary-phase points of $D_{l}^{(p)}(\theta)$ is graphed as a function of scattering angle for one internal reflection, $p$ $=2$. The result is compared with the ray theory prediction for the incident-ray impact parameter as a function of scattering angle obtained by using Eq. (1). Again the correspondence with the geometrical rays is near perfect. Also graphed is the prediction of Eq. (36) for the partialwave number of the $j=2$ tunneling-ray stationary-phase point. As was the case for $p=1$, the correspondence for the tunneling ray is again very good. Scattering radiation associated with the complex ray of the rainbow ${ }^{29}$ propagates into the shadow region $\mathrm{C}$ to the left of the merge point of the two geometrical rays in the figure (i.e., to smaller scattering angles), and radiation is shed by electromagnetic surface waves into the shadow region $\mathrm{S}$ to the right of the merge point of the larger impactparameter geometrical ray and the tunneling ray (i.e., to larger scattering angles). As was seen in Eq. (36), the $p=2$ tunneling ray in Fig. 7 extends over a farther angular interval for $\Theta<\Theta_{c}^{p}$ than does the $p=1$ tunneling

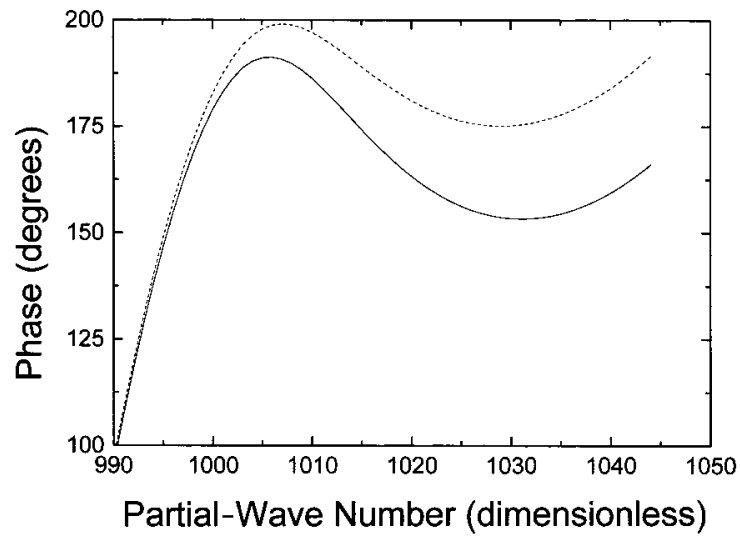

(a)

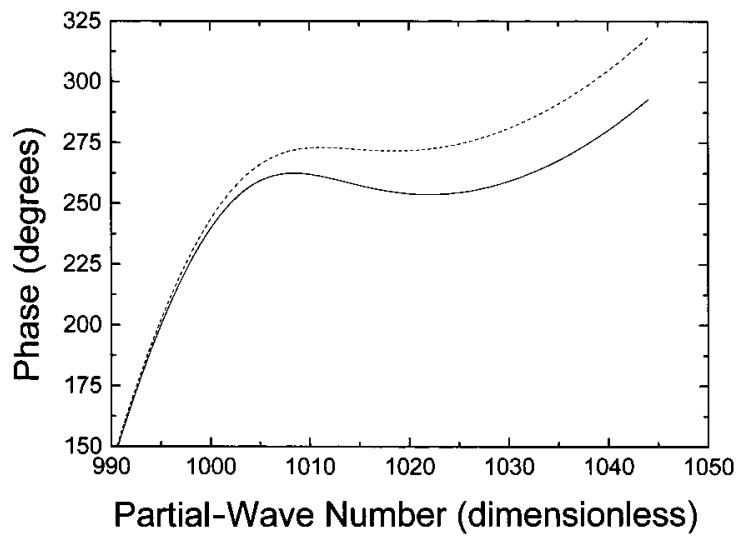

(b)

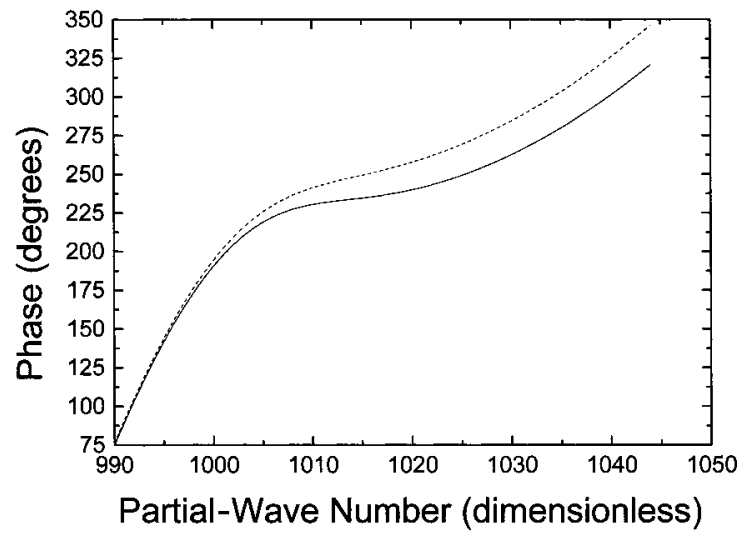

(c)

Fig. 6. Phase of Eq. (51) as a function of partial-wave number for $p=1, x=1000.0, n=1.333$, and $j=1$ (solid curve) and $j=2$ (dashed curve) for (a) $\theta=79.5^{\circ}$, (b) $\theta=81.0^{\circ}$, and (c) $\theta=82.75^{\circ}$ illustrating the merging of the geometrical- and tunneling-ray stationary-phase points. 


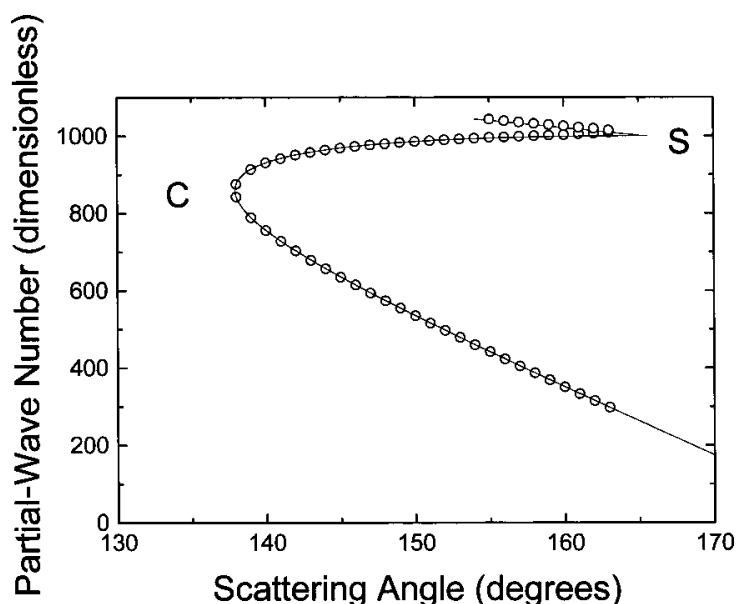

Fig. 7. Partial-wave number of the $p=2$ stationary-phase points of Eq. (51) as a function of scattering angle for $x$ $=1000.0, n=1.333$, and $j=2$ (circles). The curved solid line is the prediction of ray theory, and the straight slanted solid line at the top right of the figure is the prediction of Eq. (36) for the tunneling ray. Region $\mathrm{C}$ contains radiation associated with the complex ray on the shadow side of the rainbow, and region $\mathrm{S}$ contains radiation shed by electromagnetic surface waves on the shadow side of the critical scattering angle.

ray in Fig. 5. As a more extreme case for a larger number of internal reflections, Fig. 2 of Ref. 9 indicates that the $p=12$ tunneling ray most likely extends all the way back to the $p=12$ rainbow angle.

One last concern about the tunneling ray is that if the Fock transition is in fact a two-ray-to-zero-ray transition, why can't the interference between the geometrical ray and the tunneling ray be seen in the two-ray region of Fig. 2 ? The answer is that interference is not evident because, owing to the large damping in relations (37) and (50) produced by tunneling through the centrifugal barrier and back, the tunneling ray is extremely weak compared with the geometrical ray it interferes with. As an example, consider $x=1000.0$ and $n=1.333$, and assume $p=1$ so that the damping factors in relation (37) are as small as possible for this value of $x$ and $n$. As mentioned previously, the $p=1$ critical scattering angle is $\theta_{c}^{1}=82.79^{\circ}$. For $\theta=79.0^{\circ}, 79.5^{\circ}, 80.0^{\circ}$, and $80.5^{\circ}$, the ratio of the tunneling-ray amplitude in relation (37) to that of the geometrical ray is $0.0032,0.0222,0.1388$, and 0.7695 , respectively. For $\theta \leqq 80.0^{\circ}$, the modulation depth of the interference is small. But as $\theta$ approaches $\theta_{c}^{1}$, although the two component amplitudes become comparable, one is now sufficiently close to the merge point of the two rays that the stationary-phase calculation leading to relation (37) becomes invalid. To make matters worse, the phase of the tunneling-ray amplitude in relation (37) changes by only $35^{\circ}$ for $79.0^{\circ} \leqslant \theta \leqslant 80.5^{\circ}$, giving rise to a very long angular periodicity of any interference that may be occurring. For a smaller sphere the exponential damping factor decreases and the interference is possibly stronger. But one is now farther from the semiclassical limit, and many other corrections that we previously ignored must be added to the analysis. Thus it would appear that under most circumstances, interference between the geometrical ray and the tunneling ray in the lit region is very meager at best.
When one examines the Fourier transform of the scattered intensity, the contribution of weak rays often becomes apparent, since in Fourier transform space these rays no longer overlap with and are dominated by the contribution of much stronger rays. But the weak glare point $^{3,30-32}$ associated with the tunneling ray is easily lost in the finite-aperture ringing structure accompanying the much larger geometrical-ray glare point since the partialwave numbers of the geometrical ray and the tunneling ray in Figs. 4, 5, and 7 are close together. Also, for a spherical water droplet, the $p=12,14$ rainbows $^{33}$ occur near the $p=1$ critical scattering angle, further complicating the glare-point pattern. Thus the tunneling ray may not be apparent in the Fourier transform of the scattered intensity sampled in the vicinity of the critical scattering angle for scattering by a sphere. A similar transition, however, occurs for scattering by a spheroid, and it remains an open question as to whether the appearance of the superweak caustic in spheroid scattering is any more favorable to observation than it is in sphere scattering.

\section{DISCUSSION}

In spite of the improbability of detecting effects associated with the tunneling-ray-geometrical-ray coalescence in light-scattering experiments, the two-ray-to-zero-ray interpretation of the Fock transition is nonetheless pleasing and appealing on theoretical grounds. In light scattering by small particles, many different transitions between lit and shadow regions have been identified. Those associated with caustics require the change of an even number of contributing rays when the observation point crosses the transition, and these transitions are accompanied by a strong interference pattern in the lit region. The transitions associated with weak caustics require no change in the number of contributing rays when the observation point crosses the transition, and they are accompanied by a much weaker interference pattern in the lit region that is produced by diffractive effects. At the level of the $(x / 2)^{1 / 3}$ contribution to the phase $Y$ in relation $(24)$, the Fock transition between the lit and the shadow region for transmission of near-grazing incident rays following $p$ -1 internal reflections is fundamentally different from these other transitions since it exhibits a change of one contributing ray when the observation point crosses the transition. Understanding the reason for this fundamental difference is what motivated this research.

The nature of the transition changes with the inclusion of the smaller $(x / 2)^{-1 / 3}$ contribution to the phase of $Y$ in relation (24). At this higher level of approximation, the far-zone Fock transition in light scattering by a dielectric sphere can be interpreted as a two-ray-to-zero-ray superweak caustic accompanied by an exceedingly weak interference pattern in the lit region. The reason that it previously appeared as if the Fock transition were a one-rayto-zero-ray transition is that (1) in the context of ray theory, there is only one geometrical ray that strikes the sphere and is refracted through it and (2) in the context of wave theory only the first substantive correction to the ray theory phase was included in previous analytical approximations. The significance of the contribution of the 
tunneling ray in near-critical-angle scattering by a dielectric sphere is that it provides additional evidence for a universality in the method by which rays extinguish themselves when the observer goes from a lit region to a shadow region. Setting aside diffractive effects, rays extinguish themselves in pairs. This last statement is well known, but the results derived here demonstrate that it applies to a larger class of lit-to-shadow transitions than is usually thought.

\section{REFERENCES AND NOTES}

1. M. V. Berry and C. Upstill, "Catastrophe optics: morphologies of caustics and their diffraction patterns," Prog. Opt. 18, 257-346 (1980).

2. P. L. Marston and E. H. Trinh, "Hyperbolic umbilic diffraction catastrophe and rainbow scattering from spheroidal drops," Nature 312, 529-531 (1984).

3. J. F. Nye, "Rainbow scattering from spheroidal drops-an explanation of the hyperbolic umbilic foci," Nature $\mathbf{3 1 2}$, 531-532 (1984).

4. N. Fiedler-Ferrari, H. M. Nussenzveig, and W. J. Wiscombe, "Theory of near-critical-angle scattering from a curved interface," Phys. Rev. A 43, 1005-1038 (1991).

5. P. L. Marston, "Critical angle scattering by a bubble: physical-optics approximation and observations," J. Opt. Soc. Am. 69, 1205-1211 (1979).

6. H. M. Nussenzveig, "High-frequency scattering by a transparent sphere. I. Direct reflection and transmission," J. Math. Phys. 10, 82-124 (1969).

7. H. M. Nussenzveig, "High-frequency scattering by an impenetrable sphere," Ann. Phys. (New York) 34, 23-95 (1965).

8. J. A. Lock and E. A. Hovenac, "Diffraction of a Gaussian beam by a spherical obstacle," Am. J. Phys. 61, 698-707 (1993).

9. The interpretation of the second stationary-phase point in terms of the tunneling ray was briefly discussed in J. A Lock, "Cooperative effects among partial waves in Mie scattering," J. Opt. Soc. Am. A 5, 2032-2044 (1988).

10. H. C. van de Hulst, Light Scattering by Small Particles (Dover, New York, 1981), pp. 203-206.

11. E. Hecht, Optics, 2nd ed. (Addison-Wesley, Reading Mass., 1987), pp. 94-96.

12. Ref. 10, pp. 206-207.

13. Ref. 10, pp. 119-126.

14. E. A. Hovenac and J. A. Lock, "Assessing the contributions of surface waves and complex rays to far-field Mie scattering by use of the Debye series," J. Opt. Soc. Am. A 9, 781795 (1992).

15. Ref. 10, pp. 208-209.

16. M. Abramowitz and I. A. Stegun, eds., Handbook of Mathematical Functions (National Bureau of Standards, Washington D.C., 1964), pp. 366-367, Eqs. (9.3.15, 9.3.16, 9.3.19, 9.3.20, 9.3.23, 9.3.24, 9.3.27, 9.3.28)

17. Ref. 16, p. 446.

18. Ref. 10 , p. 213.

19. K. W. Ford and J. A. Wheeler, "Semiclassical description of scattering," Ann. Phys. (N.Y.) 7, 259-286 (1959).

20. Ref. 10, p. 212.

21. M. V. Berry and K. E. Mount, "Semiclassical approximations in wave mechanics," Rep. Prog. Phys. 35, 315-397 (1972), eq. (6.12).

22. H. M. Nussenzveig, "High-frequency scattering by a transparent sphere. II. Theory of the rainbow and the glory," J. Math. Phys. 10, 125-176 (1969), Figs. 7, 10.

23. Ref. 16, p. 446, Eq. (10.4.9), and pp. 448-449, Eqs. (10.4.60, 10.4.64).

24. Ref. 16, p. 478, Table 10.13 .

25. Ref. 16, p. 449, Eq. (10.4.63).

26. A. W. Snyder and J. D. Love, "Reflection at a curved dielectric interface-electromagnetic tunneling," IEEE Trans. Microwave Theory Tech. MTT-23, 134-141 (1975). It should be noted that Figs. 3b and 3c in Ref. 8 are in error in that the incident ray should strike the centrifugal barrier tangentially and then tunnel radially to the sphere surface.

27. H. M. Nussenzveig, "Tunneling effects in diffractive scattering and resonance," Comments At. Mol. Phys. 23, 175-187 (1989).

28. B. R. Johnson, "Theory of morphology-dependent resonances: shape resonances and width formulas," J. Opt. Soc. Am. A 10, 343-352 (1993).

29. H. M. Nussenzveig, "Complex angular momentum theory of the rainbow and the glory," J. Opt. Soc. Am. 69, 1068-1079, 1193-1194 (1979), Fig. 2.

30. J. A. Lock, "Theory of the observations made of high-order rainbows from a single water droplet," Appl. Opt. 26, 52915298 (1987).

31. H. C. van de Hulst and R. T. Wang, "Glare points," Appl. Opt. 30, 4755-4763 (1991).

32. P. L. Marston, "Cusp diffraction catastrophe from spheroids: generalized rainbows and inverse scattering," Opt. Lett. 10, 588-590 (1985).

33. J. D. Walker, "Multiple rainbows from single drops of water and other liquids," Am. J. Phys. 44, 421-433 (1976). 